\title{
Validez de la Estructura Interna del Utrecht Work Engagement Scale (UWES) en trabajadores peruanos
}

\section{Internal structure validity of Utrecht Work Engagement Scale (UWES)}

\section{in Peruvian workers}

\author{
César Merino-Soto ${ }^{1}$, Manuel Fernández-Arata ${ }^{2}$ y Arturo Juárez-García ${ }^{3}$ \\ ${ }^{1}$ Psicólogo. Investigador externo en el Instituto de Investigación en Psicología, Universidad de \\ San Martín de Porres, Lima, Perú. E-mail: cmerinos@usmp.pe \\ ${ }^{2}$ Psicólogo. Director del Instituto de Investigación de la Escuela Profesional de Psicología de la \\ Universidad de San Martín de Porres, Lima, Perú. E-mail: mfernandeza1@usmp.pe \\ ${ }^{3}$ Psicólogo. Profesor-investigador de tiempo completo en el Centro de Investigación \\ Transdisciplinar en Psicología de la Universidad Autónoma del Estado de Morelos, México. \\ E-mail: arturojuarezg@hotmail.com
}

Los autores agradecen a los participantes del estudio, así como a las autoridades institucionales que permitieron el acceso.

Instituto de Investigación en Psicología, Universidad de San Martín de Porres. Lima, Perú.

\section{Resumen}

\section{El Utrecht Work Engagement Scale} (UWES), medida de engagement laboral, ha tenido controversias respecto a la interpretación de su dimensionalidad, es decir, si sus puntajes pueden ser unidimensionales, multidimensionales o de dos niveles (unidimensional y multidimensional). El presente estudio tuvo por objetivo examinar la estructura interna (dimensionalidad, invarianza de medición y confiabilidad) del UWES (dos versiones: larga y breve) en una muestra de 636 trabajadores peruanos. Se aplicó el modelamiento de ecuaciones estructurales para la evaluación de tres modelos de dimensionalidad: un solo factor (unidimensionalidad), tres factores relacionados (multidimensionalidad) y bifactor (dos niveles de interpretación: factor general y factores específicos). Los resultados indican que, luego de aplicar el modelamiento bifactor, la varianza común obtenida de un factor general es más fuerte que la interpretación de tres dimensiones. Este resultado se repitió en ambas versiones del UWES (larga y breve). La invarianza de medición fue satisfactoria en el nivel configuracional, métrica y escalar (en ambas versiones). Con respecto a la consistencia interna, se obtuvieron coeficientes satisfactorios (mayores a .70). Se concluye que el modelo de tres dimensiones del UWES para obtener los puntajes debe ser reemplazado por un modelo unidimensional, representado con un puntaje total. Se discuten las implicaciones teóricas y prácticas.

Palabras clave: engagement, modelamiento de ecuaciones estructurales, validez, trabajadores, invarianza de medición.

\section{Abstract}

The Utrecht Work Engagement Scale (UWES), a measure of work engagement, has had controversies regarding the interpretation 
of its dimensionality, that is, whether its scores can be one-dimensional, multidimensional or two-level (one-dimensional and multidimensional). The dimensionality of the UWES has been questioned mainly due to the high covariation between its components, an aspect consistently verified in the observed scores and latent variables (De Bruin y Henn 2013), even by the same authors (Schaufeli et al., 2006). This has two implications, one operational and one conceptual. In the operational context, the calculation of scores is also influenced by the statistical differentiation of the factors found in the factor analyzes. This means that if one or more factors are retained and justified, this also determines the same number of observed scores that can be calculated and interpreted. On the other hand, the conceptual implication is that the comprehensive framework of a three-dimensional psychological phenomenon differs from the one-dimensional. For example, the differentiation between dimensions generates the possibility of different trends between dimensions (e.g., one of three is high and the other low) and in this case, the concept of engagement becomes very generic and ambiguous to name and understand the different possibilities. Specific combination of its dimensions or jeopardizes its content validity, implying the need for other more specific concepts, which although related to engagement, can be independent.

The objective of the present study was to examine the internal structure (dimensionality, measurement invariance and reliability) of the UWES (two versions: long and short) in a sample of 636 Peruvian workers. Structural equation modeling was applied to evaluate three dimensionality models: a single factor (one-dimensionality), three related factors (multidimensionality) and bifactor (two levels of interpretation: general factor and specific factors). The results indicate that, after applying bifactor modeling, the common variance obtained from a general factor is stronger than the three-dimensional interpretation. This result was repeated in both versions of the UWES (long and short). The measurement invariance was satisfactory at the configurational, metric and scalar levels (in both versions). Regarding internal consistency, satisfactory coefficients (greater than .70) were obtained.

Three issues emerge from this study that modify the original theoretical interpretation of the UWES (which consists of using three related factors). The first is the existence of a general factor underlying the items, and which is statistically substantial as a source of variance of the items, independent of specific factors. In this general factor, the items generally contribute to their variance, except for two items whose discriminative capacity is moderately low (11 and 13, both of the specific factor Absorption). The main element of this general factor is the Dedication factor, whose items completely represented this general factor. Second, the differentiation of two specific factors corresponding to Vigor and Absorption does not appear to be psychometrically sufficient to describe nested constructs within the engagement construct, and therefore its independent interpretation of the general factor could be questioned. However, a contrary argument is that both factors show different amount of specific variance, although low compared to the general factor; for example, Absorption shows more divergent validity compared to Vigor, while the latter contains more common variance (general factor). Third, Dedication was completely absorbed by the general factor, and is only relevant insofar as its items are completely related to the general factor. Therefore, this component lost statistical autonomy and, consequently, very poor conceptual differentiation. It is concluded that the UWES - three-dimensional model to obtain the scores must be replaced by a one-dimensional model, represented with a total score. Implications are future research are discussed.

Keywords: engagement, structural equations modeling, validity, workers, measurement invariance. 


\section{Introducción}

El engagement es un estado psicológico de naturaleza positiva, antagónico al burnout y, de acuerdo al modelo de Schaufeli y Bakker (2010a), está conformado por un componente conductual (vigor), un componente emocional (dedicación) y uno cognitivo (absorción). El engagement ha sido popular en la salud ocupacional y en el campo de los Recursos Humanos. Su antigua denominación, "work engagement", se convierte más tarde en "engaged employee" (Schaufeli, Shimazu, Hakanen, Salanova y De Witte, 2017) y en Latinoamérica como "entusiasmo laboral" (Juárez et al., 2015). El engagement laboral es definido como un estado de ánimo positivo, satisfactorio y relacionado con el trabajo que se caracteriza por el vigor, la dedicación y la absorción del trabajador hacia su actividad laboral (Schaufeli y Bakker, 2010a, 2010b) y, de acuerdo a estos autores, el vigor se caracteriza por tener altos niveles de energía y resiliencia mental en el trabajo; la dedicación, por tener un fuerte nivel de involucramiento con el trabajo y experimentar entusiasmo, inspiración, orgullo y desafío; y la absorción se refiere al estado de completa atención y concentración que hace sentir como que el tiempo avanza rápidamente, sin darse cuenta de ello. Estas características, en conjunto, describen la forma en la que los trabajadores experimentan su trabajo, si lo consideran estimulante para invertir energías personales, tiempo y esfuerzo (vigor), si es algo significativo, y si lo absorbe tanto como para estar completamente concentrado en él (Bakker, Albrecht y Leiter, 2011). La actitud positiva de los empleados comprometidos crea sus propios comentarios positivos en términos de apreciación, reconocimiento y éxito (Bakker et al., 2011), a tal punto que, aunque se sienten cansados después de un largo día de duro trabajo, describen su cansancio como un estado placentero que está asociado con logros positivos y actividades divertidas.

Para la medición del engagement, el autoinforme más utilizado es el Utrecht Work Engagement Scale (UWES), con el que se han realizado estudios en más de trece países (Salanova y Llorens, 2008). El modelo Demandas Recursos Laborales (JD-R) fue el marco conceptual para investigar la validez de contenido del UWES, dado que describe algunos componentes de un proceso motivacional estimulado por la presencia de recursos laborales positivos (Salanova y Llorens, 2008; Schaufeli et al., 2017), que promueven los esfuerzos para realizar sus tareas laborales e induce a un estado de entusiasmo por el trabajo. Las investigaciones previas muestran consistentemente que los recursos laborales y personales son importantes predictores del entusiasmo laboral (Bakker et al., 2011).

La propuesta original del UWES estuvo constituida por 24 ítems; posteriormente, una revisión por parte de los autores lo redujo a 17 ítems (Schaufeli et al., 2002) que miden las dimensiones de vigor (seis ítems), dedicación (cinco ítems) y absorción (seis ítems). Sus cualidades psicométricas han sido demostradas en varios estudios de validez de constructo y de consistencia interna (Schaufeli et al., 2010). Luego, utilizando una base de datos internacional de 10 países, Schaufeli, Bakker y Salanova (2006) elaboraron una versión breve de nueve ítems, con tres ítems para cada una de las dimensiones del instrumento original. Aunque el modelo de tres factores se ajustó satisfactoriamente, un modelo unidimensional también lo hizo de manera satisfactoria, pero las correlaciones latentes entre los factores (vigor, dedicación y absorción) fueron muy altas en todas las muestras examinadas. Un aporte adicional fue la obtención de una versión breve de nueve ítems, la cual se elaboró teniendo en cuenta la validez aparente, el razonamiento teórico y los comentarios de los participantes del estudio (Schaufeli et al., 2006).

La dimensionalidad del UWES ha sido cuestionada principalmente por la alta covariación entre sus componentes, un aspecto verificado consistentemente en las correla- 
ciones entre los puntajes observados y entre sus correspondientes variables latentes (De Bruin y Henn 2013), incluso por los mismos autores (Schaufeli et al., 2006). No es claro qué magnitud correlacional se considera alta en la literatura estadística debido a las diferentes opiniones al respecto, pero usualmente en ciencias sociales las correlaciones altas se identifican como iguales o mayores a .50 (Cohen, 1992). Este patrón correlacional entre las escalas del UWES no ha variado prácticamente en los estudios publicados en habla hispana y en el resto de los países. Por ejemplo, en los estudios psicométricos o no psicométricos realizados en sujetos latinoamericanos, las correlaciones entre sus subescalas son de alrededor de .50 o más (Christian y Slaughter, 2007; Flores et al., 2015; Fong, 2012; Hernández, Llorens y Dickinson, 2016; JuárezGarcía et al., 2015; Müller, Pérez y Ramírez, 2013; Rodríguez-Montalbán, Martínez-Luyo y Sánchez-Cardona, 2014; Sonnentag, 2003; Spontón, Medrano, Maffei, Spontón y Castellano, 2012; Spontón, Trógolo, Castellano y Medrano, 2019; Vecina, Chacon, Sueiro y Barron, 2012; Wefald y Downey, 2009). Este patrón correlacional tampoco es extraño en estudios con participantes no hispanos (e. g., Agarwal, 2014; Chughtai y Buckley, 2013; Petrović, Vukelić y Čizmić, 2017; Sinval, Pasian, Queirós y Marôco, 2018; Vallières, McAuliffe, Hyland, Galligan y Ghee, 2017). Estos hallazgos señalan que, generalmente, el problema ocurre con independencia del contexto cultural de los participantes y que su interpretación y análisis debe considerar esta fuerza correlacional como hipótesis de trabajo.

Esto tiene dos implicancias: una operacional y otra conceptual. En el contexto operacional, el cálculo de puntajes se ve también influenciado por la diferenciación estadística de los factores hallados en los análisis factoriales, es decir que, si se retienen y justifican uno o más factores, esto determina también la misma cantidad de puntajes observados que se pueden calcular e interpretar. Por ejemplo, una decisión analítica que puede llevar a confusión es que, al mismo tiempo que se acepta su estructura tridimensional, se puede también sugerir un puntaje total del UWES con la suma simple de todos los ítems (Schaufeli y Bakker, 2003), pero sin la verificación directa. Por otro lado, la implicancia conceptual es que el marco comprensivo de un fenómeno psicológico tridimensional es diferente al unidimensional. Por ejemplo, la diferenciación entre dimensiones genera la posibilidad de tendencias distintas entre dimensiones (e. g., una de tres es alta y las otras, bajas) $\mathrm{y}$ en este caso, el concepto de engagement se vuelve muy genérico y ambiguo para nombrar y comprender las distintas posibilidades específicas de combinación entre sus dimensiones, o pone en riesgo su validez de contenido, lo cual implica la necesidad de otros conceptos más específicos que, aunque relacionados con el engagement, pueden ser independientes.

Parece que la evidencia sobre las características estructurales del UWES aún no ha alcanzado una clara representación empírica, dado que los modelos de medición examinados no han abordado explícitamente la alta covariación entre sus factores. Asimismo, la generalización de estos resultados no ha sido contrastada al contexto específico de los participantes. Adicionalmente, y de acuerdo con los resultados de De Bruin y Henn (2013), es plausible que el modelo de un factor global sea sustancialmente generalizable para explicar una parte de la varianza de los ítems del UWES, obtenido mediante la incorporación de un análisis factorial bifactor, que consiste en definir una variable latente común a todos los ítems de una escala multidimensional para identificar la varianza correspondiente a los factores específicos (asociados a los puntajes de las subescalas) y al factor general (asociado al puntaje total). De este modo se valora la estructura del constructo y la capacidad de los puntajes para arrojar información confiable (Reise, 2012; Reise, Scheines, Widaman y Haviland, 2013; Rodriguez, Reise y Haviland, 2016a, 2016b). En población de hispanos no 
inmigrantes, hasta la fecha no se reportado la evaluación de este modelo $\mathrm{y}$, dada las correlaciones altas halladas en los estudios precedentes, es probable que la descomposición de la varianza compartida entre los factores del UWES pueda revelar un patrón similar al estudio de De Bruin y Henn. Así que, examinarlo en el contexto de otros probables modelos arrojaría nueva información sobre la naturaleza de la experiencia de engagement en trabajadores de habla hispana y, específicamente, de Perú. El objetivo del presente estudio es examinar la validez de la estructura interna del UWES, tomando en cuenta el modelo de medición que mejor pueda representar las relaciones internas de los ítems con sus constructos y de estos entre sí, y valorándolo desde la aplicación del modelo bifactor.

De acuerdo con los hallazgos sobre el tamaño predominantemente grande de las correlaciones entre las dimensiones del UWES en diferentes estudios, y los resultados de De Bruin y Henn (2013), se planteó la siguiente hipótesis de estructura interna: Un modelo de medición que incluya una dimensión general y dimensiones específicas (i. e., bifactor) representará mejor las respuestas al UWES comparado con el modelo con dimensiones específicas correlacionadas.

\section{Método}

\section{Participantes}

La población de estudio estuvo compuesta por trabajadores peruanos con contrato formal. La muestra participante fue de tres grupos: dos grupos homogéneos de trabajadores (docentes de educación regular y empleados de mantenimiento) y otro grupo heterogéneo de trabajadores. Se accedió a los participantes mediante el contacto con sus organizaciones de trabajo, ubicadas en Lima o fuera de Lima. Las organizaciones participantes fueron seleccionadas incidentalmente, dentro de un marco no probabilístico. La muestra elegible cumplió con los criterios de inclu- sión: (a) nacionalidad peruana, (b) actividad laboral actual, (c) experiencia mínima de un año, y (d) consentir participar voluntariamente en el estudio. Los participantes seleccionados fueron 636 trabajadores $(39.6 \%$ mujeres; un sujeto sin dato), de los cuales el $59.6 \%$ eran docentes, el $23.9 \%$, empleados de mantenimiento y un $16.5 \%$ de otras profesiones como abogados, ingenieros, médicos, enfermeros, entre otras. La distribución de sujetos fue similar en entidades públicas (351, $55.2 \%)$ y privadas $(260,40.9 \%$; sin información: $25,3.9 \%$ ). En cuanto al estado civil, el $55.9 \%$ eran casados o convivientes, mientras que el resto eran solteros $(37.1 \%)$ o separados (4.6\%), y de un $2.3 \%$ no se obtuvo información. El 54 \% había nacido en Lima (sin información, $3 \%$ ), y el resto fuera de Lima. La edad promedio fue 41.09 años (DE = 11.67, $\operatorname{Min}=19$, Max $=69$ ); existieron diferencias entre hombres y mujeres con respecto a la edad $(\mathrm{t}[626]=2.61, \mathrm{p}<.01)$ y años de experiencia laboral $(\mathrm{t}[624]=2.23, \mathrm{p}<.05)$, pero ambos de magnitud pequeña $(\mathrm{d}=.21 \mathrm{y}$ .18 , respectivamente). La experiencia laboral en el grupo total varió entre 0 y 45 años $(\mathrm{M}=$ 13.9, $\mathrm{DE}=1.65)$. Existieron leves diferencias distribucionales de acuerdo al sexo en el tipo de ocupación $\left(\chi^{2}(2)=36.9 ; \mathrm{p}<.01\right.$, V Cramer $=.24$ ), en que el número de mujeres predominaba entre los educadores.

\section{Instrumentos}

\section{Utrecht Work Engagement Scale (UWES, Salanova et al., 2000; Schaufeli et al., 2006)}

Se utilizó la versión de 15 ítems (Salanova et al., 2000) y de nueve ítems (Schaufeli et al., 2006); ambas están conformadas por tres dimensiones: Vigor (VI, cinco ítems), Dedicación (DE, cinco ítems) y Absorción ( $\mathrm{AB}$, cinco ítems). Cada subescala de la versión UWES-9 está constituida por tres ítems de la versión UWES-15. La puntuación de todos los ítems es una escala ordinal de siete puntos, desde 0 (nunca) hasta 6 puntos (diariamente). Existe 
un estudio con maestros peruanos (Flores, Fernández, Juárez, Merino y Guimet, 2015) en que se replicó satisfactoriamente la estructura de tres dimensiones en ambas versiones, y la consistencia interna fue: VI (alrededor de .78), DE (alrededor de .79) y AB (alrededor de .64).

\section{Procedimiento}

\section{Recolección de datos}

Se realizó en los lugares de trabajo de los participantes, y se aplicó el UWES junto a otras que forman parte de una investigación mayor, presentadas en el siguiente orden: formulario de consentimiento informado, UWES y el resto de los instrumentos. Se solicitó autorización a las autoridades principales de los centros educativos, a la sección de posgrado de una universidad privada para evaluar a sus estudiantes de Maestría y a los directivos de una empresa para evaluar a los empleados de mantenimiento. En todos los casos se dieron instrucciones estandarizadas, mediante las cuales se les informó sobre los objetivos del estudio, la actitud honesta para responder, la confidencialidad de las respuestas mediante el anonimato de la participación y de los resultados respecto a la organización, y la posibilidad de no continuar respondiendo si así lo quisieran. Luego de ello, los participantes firmaron el consentimiento informado en el que aceptaban su participación voluntaria. El desarrollo de los procedimientos de recolección de datos se acomodó al Código Ético y Deontológico del Psicólogo en el Perú (Colegio de Psicólogos del Perú, 2004) $\mathrm{y}$ al Informe Belmont (United States, 1978), con relación a la protección de la identidad, el respeto, el buen nombre, la participación voluntaria y los fines estrictamente académico-investigativos de los resultados de investigación.

\section{Análisis}

Se hizo un análisis de los ítems mediante los estadísticos descriptivos usuales. Algunos de estos se estandarizaron para mejorar la inter- pretación: para la variabilidad se usó el índice de variación ordinal (IVO; Berry y Mielke, 1994) y para la asimetría, el índice estandarizado de asimetría SSI (standardized skew index; Malgady, 2007). En ambos métodos, los valores cerca de 1 indican el incremento del atributo estadístico examinado. La bimodalidad se evaluó mediante el índice BC (SAS Institute, 2012), en el que valores superiores a .55 indican potencialmente distribuciones bimodales (Freeman y Dale, 2013).

La estructura dimensional se verificó mediante el modelamiento de ecuaciones estructurales, específicamente con un análisis factorial confirmatorio (CFA) para probar diferentes modelos de medición del UWES. Se hicieron algunas presunciones para este análisis: primero, que las relaciones entre los ítems pueden ser parsimoniosamente representadas por relaciones lineales bajo la normalidad bivariada. Segundo, dado que los ítems fueron construidos con el número de opciones mayor a 5, pueden aceptarse como aproximaciones suficientes de variables continuas (Bollen y Barb, 1981); por lo tanto, se usó la matriz de covarianzas entre los ítems. Tercero, se tuvo en cuenta que las respuestas al UWES tienden a producir distribuciones asimétricas; por lo tanto, se aplicó el método de máxima verosimilitud con la corrección de Satorra y Bentler (1994), SB- $\chi^{2}$, para así controlar el efecto de la no normalidad multivariada de los ítems. La evaluación de los modelos se hizo mediante índices prácticos de ajuste, como CFI $(\geq .95)$, RMSEA $(\leq .05)$ y SRMR $(\leq .05)$. Complementariamente, se usó el criterio de información de Akaike (AIC), cuyas magnitudes comparativamente más bajas entre los modelos indican un mejor ajuste.

El análisis CFA inició con la especificación de errores no correlacionados entre los ítems y los ítems relacionados únicamente con el factor esperado, lo que significa que sus parámetros fueron estimados libremente y constreñidos a cero en los otros factores. Se fijó la varianza de cada factor en 1, para permitir evaluar la parametrización directa en 
cada ítem (Lubke y Muthén, 2004). Luego de verificar el modelo de medición apropiado del UWES, se examinó la invarianza de medición (IM) de los parámetros obtenidos mediante el procedimiento acumulativo de evaluar si el modelo cumplía varios niveles de invarianza (Meredith, 1993): configuracional (igualdad de número de constructos), débil (igualdad de cargas factoriales), fuerte (igualdad de interceptos de los ítems). Los grupos comparados para la IM fueron docentes versus no docentes, que fueron elegidos como más relevantes para examinar el impacto del tipo de trabajo sobre la propiedad de invarianza psicométrica. Se aplicó la diferencia entre CFI $\left(\Delta_{\mathrm{CFI}} \leq .01\right.$; Cheung y Resvold, 2002) como fuente principal de evaluación de IM, dado que la diferencia $\mathrm{SB}-\chi^{2}$ es sensible al tamaño muestral.

La precisión de los puntajes se evaluó con la consistencia interna, basada en el modelamiento lineal, mediante el coeficiente $\omega$ (McDonald, 1999); su variabilidad poblacional fue obtenida mediante intervalos al $95 \%$ de confianza con el método boostrap acelerado y corregido por sesgo (Kelley y Pornprasertmanit, 2016). Los programas estadísticos utilizados fueron: para el análisis del modelamiento, EQS 6.2 (Bentler y Wu, 2012) y bifactor índices calculator (Dueber, 2017); para los estadísticos en el nivel de los ítems, SPSS 26 y cálculo manual (para SSI e índice BC); para el coeficiente IVO, sg 59 (Goldstein, 1996), y para los intervalos de confianza de los coeficientes de confiabilidad, el paquete R MBESS (Kelley, 2020).

\section{Resultados}

El objetivo principal del presente estudio fue evaluar la estructura interna del UWES, para establecer la dimensionalidad, las relaciones entre los ítems y sus constructos, y la invarianza de medición respecto a dos amplios grupos laborales (i. e., docentes y no docentes). Complementariamente, fue realizado un análisis de ítems.

\section{Análisis de ítems}

Se detectaron pocos valores perdidos, entre $2(.3 \%)$ y $6(.9 \%)$ para cada ítem, y este patrón fue reconocido como aleatorio; como consecuencia, para maximizar la potencia estadística de los análisis, se imputaron los valores pedidos por la respuesta modal (opción 5). Los estadísticos descriptivos a nivel de los ítems se encuentran en la Tabla 1, desde la cual los ítems se caracterizan por mostrar una intensidad de respuesta entre las opciones: frecuentemente: una vez por semana (calificación 4) y muy frecuentemente: varias veces por semana (calificación 5). Esto se relaciona con una clara tendencia a la asimetría negativa en todos los ítems que, sin embargo, tuvo un amplio rango de acuerdo a la variabilidad del SSI. Las respuestas cubrieron generalmente todo el rango de opciones y la variabilidad fue moderadamente similar según el coeficiente IVO. La mayor variación ocurrió en el ítem 11, que tuvo el mayor índice BC. Todos los ítems mostraron distribuciones aparentemente bimodales (BC alrededor de .55), lo que se atribuye a la predominancia de respuesta en alguna de las categorías adyacentes a la respuesta con mayor frecuencia. Finalmente, la relación bivariada normal entre los ítems fue examinada con la prueba $\chi^{2}(\mathrm{gl}=4)$ de Doornik-Hansen (2008) que varió entre 309 y .793 (todos $p<.001)$, lo que indica que esta característica, claramente, no se cumple. Sin embargo, una razonable aproximación a esta condición fue satisfactoria luego de aplicar la prueba RMSEA de cercano ajuste, con la cual las relaciones bivariadas estuvieron entre .094 y $.00($ RMSEA mediana $=.043 ; 67 \%$ igual o debajo de RMSEA = .05). Finalmente, al examinar los estadísticos distribucionales multivariados (Mardia, 1970) de asimetría $\left(b_{1, k}=55.082\right)$ y curtosis $\left(b_{2, k}=403.573\right)$, estos fueron grandes y estadísticamente significativos: $\chi^{2}(680)=5878.88$ y $\chi^{2}(1)=6892.73$, respectivamente. La prueba multivariada Doornik-Hansen (2008) también confirmó este resultado: $\chi^{2}(30)=2849.574(\mathrm{p}<.01)$. 


\section{Tabla 1}

Estadísticos descriptivos para los items.

\begin{tabular}{c|c|c|c|c|c|c|c|c|c} 
& & \multicolumn{9}{|c|}{ Indicadores de variabilidad } & \multicolumn{3}{|c}{ Indicadores distribucionales } \\
& & Min & Max & DE & IVO & $\mathbf{g}_{1}$ & SSI & $\mathbf{g}_{2}$ & BC \\
\hline E1 & 4.81 & 0 & 6 & 1.182 & .411 & -1.097 & -.393 & .901 & .563 \\
E2 & 4.43 & 0 & 6 & 1.349 & .478 & -.974 & -.268 & 0.65 & .532 \\
E3 & 4.86 & 0 & 6 & 1.252 & .425 & -1.326 & -.423 & 1.528 & .607 \\
E4 & 5.20 & 0 & 6 & .986 & .320 & -1.686 & -.867 & 3.971 & .550 \\
E5 & 5.13 & 0 & 6 & .935 & .313 & -1.341 & -.767 & 2.615 & .497 \\
E6 & 4.96 & 0 & 6 & 1.174 & .400 & -1.288 & -.467 & 1.585 & .578 \\
E7 & 4.98 & 0 & 6 & 1.151 & .394 & -1.244 & -.470 & 1.508 & .563 \\
E8 & 4.92 & 0 & 6 & 1.141 & .392 & -1.248 & -.479 & 1.704 & .542 \\
E9 & 5.14 & 0 & 6 & 1.037 & .343 & -1.535 & -.714 & 3.144 & .545 \\
E10 & 5.11 & 1 & 6 & 1.046 & .422 & -1.303 & -.595 & 1.405 & .610 \\
E11 & 4.16 & 0 & 6 & 1.857 & .670 & -.824 & -.119 & -.497 & .667 \\
E12 & 5.04 & 0 & 6 & 1.228 & .403 & -1.536 & -.509 & 2.118 & .655 \\
E13 & 4.35 & 0 & 6 & 1.574 & .558 & -1.033 & -.208 & .415 & .603 \\
E14 & 4.85 & 0 & 6 & 1.227 & .420 & -1.308 & -.434 & 1.893 & .552 \\
E15 & 4.70 & 0 & 6 & 1.361 & .469 & -1.259 & -.340 & 1.383 & .588
\end{tabular}

Notas: Error estándar de $\mathrm{g}_{1}$ y g $\mathrm{g}_{2}$ :097 y .193, respectivamente. M: media. IVO: índice de variación ordinal. g : coeficiente de asimetría. $\mathrm{g}_{2}$ : coeficiente de curtosis. SSI: índice estandarizado de asimetría. BC: índice de bimodalidad.

\section{Estructura interna}

\section{UWES-15}

Sobre una base a priori, se probaron varios modelos probables para la estructura latente del UWES. Se verificó primero un modelo unidimensional $\left(\mathrm{M}_{1}\right)$, en que la varianza de los ítems es explicada por una sola variable latente; en segundo lugar, el modelo de tres factores oblicuos $\left(\mathrm{M}_{2}\right)$, que expresa el planteamiento teórico original del engagement y que, principalmente, fue la hipótesis nula de estudios previos. Se evaluó un modelo bifactor completo $\left(\mathrm{M}_{3 \mathrm{a}}\right)$, que consistió en especificar dos fuentes de variación de los ítems: un factor general y tres factores específicos; finalmente, y dentro de este mismo modelo bifactor, se probó el bifactor incompleto para corroborar el reciente hallazgo de De Bruin y Henn (2013). De acuerdo a la literatura revisada (Flores et al., 2015; Schaufeli et al., $2002,2006)$, era realista esperar reespecificaciones a posteriori para mejorar el ajuste, pero estas se efectuaron priorizando la magnitud de los índices de modificación (IM) y su base teórico-racional.

El modelo unidimensional $\left(\mathrm{M}_{1}\right)$ mostró el más bajo ajuste, lo que indicó que hay otros parámetros estructurales que fueron necesarios modelar (Tabla 2). En las siguientes evaluaciones de los modelos $\left(\mathrm{M}_{2}\right.$ : oblicuo, y $\mathrm{M}_{3}$ : bifactor), se descubrió que los índices de modificación para la correlación entre 
los errores de los ítems 4 y 5,9 y 10 fueron consistentes muy elevados (especialmente entre los dos últimos). Además, en todos los modelos, sus residuales entre ellos fueron los más altos respecto al resto $(>.11)$. Sobre la base de esta observación a posteriori, se liberaron las correlaciones entre sus errores en cada modelo estimado, para así ver compara- tivamente su efecto sobre el ajuste del modelo. Sin embargo, para mantener un enfoque parsimonioso en esta reespecificación a posteriori, únicamente se liberó la covariación de errores entre los ítems 9 y 10 , pues consistentemente mostraban los índices de modificación muy altos, y teóricamente parecen estar mejor vinculados.

Tabla 2

Resultados del ajuste para UWES-15.

\begin{tabular}{|c|c|c|c|c|c|c|}
\hline UWES - 15 & SB- $\chi^{2}(g l)$ & CFI & $\begin{array}{c}\text { RMSEA } \\
(\text { I.C. } 90 \%) \\
\end{array}$ & SMSR & AIC & $\begin{array}{c}\text { Residuales } \\
\quad>|0.10| \\
\end{array}$ \\
\hline$M_{1}:$ Unidimensional & $482.472(90)$ & .853 & $.83(.76, .90)$ & .74 & 302.472 & 14 \\
\hline \multicolumn{7}{|l|}{$M_{2}:$ Factores oblicuos } \\
\hline $\mathrm{M}_{2 \mathrm{a}}:$ modelo base & $2773.613(105)$ & .928 & $.59(.51, .67)$ & .48 & 104.493 & 4 \\
\hline $\begin{array}{l}\mathrm{M}_{2 \mathrm{~b}}: \text { modelo base } \\
\text { modificado }\end{array}$ & $226.088(86)$ & .948 & $.51(.43, .59)$ & .46 & 54.089 & 3 \\
\hline$M_{3}:$ Bifactor & & & & & & \\
\hline $\mathrm{M}_{3 \mathrm{a}}$ : bifactor completo & $234.156(75)$ & .940 & $.58(.49, .66)$ & .38 & 84.156 & 3 \\
\hline $\mathrm{M}_{3 \mathrm{~b}}:$ bifactor incompleto & $243.7006(80)$ & .939 & $.57(.49, .65)$ & .38 & 83.701 & 2 \\
\hline $\begin{array}{c}\mathrm{M}_{3 \mathrm{c}}: \text { bifactor incompleto } \\
\text { modificado }\end{array}$ & $192.454(79)$ & .957 & $.48(.39, .56)$ & .35 & 34.454 & 1 \\
\hline
\end{tabular}

Notas: $M_{1}$ : unidimensional. $M_{2 a}$ : factores oblicuos. $M_{2 b}$ : factores oblicuos, modificado. $M_{3 a}$ : bifactor completo. $M_{3 b}$ : bifactor incompleto. $\mathrm{M}_{3 \mathrm{c}}$ : bifactor incompleto, modificado. UWES15: Modelo independiente: SB- $\chi^{2}(105)=2773.613$.

En el modelo de factores oblicuos, el modelo inicial $\left(\mathrm{M}_{2 \mathrm{a}}\right)$ mostró un sustancial y mejor ajuste comparado con $\mathrm{M}_{1}$ (unidimensional), y sus índices de ajuste fueron satisfactorios, excepto CFI $(<.95)$. Cuando se liberó la restricción de correlación cero entre los ítems 9 y $10(r=.445, \mathrm{Cov}=.233, z=4.10$, $\mathrm{p}<.01)$, el modelo mejoró sustancialmente $\left(\mathrm{M}_{2 \mathrm{~b}}\right)$. Aunque este resultado pudo culminar el modelamiento, las correlaciones altas (> $.50)$ o muy altas $(>.70)$ entre los factores (Tabla 3) sugirieron que se puede introducir un factor adicional para explicar la varia- ción común global de los ítems (Reise, 2012; Rodríguez et al., 2016a, 2016b). En la última evaluación, se probó el modelo bifactor $\left(\mathrm{M}_{3}\right)$ introduciendo un factor general $(\mathrm{Fg})$, en que el modelo bifactor completo $\left(\mathrm{M}_{3 \mathrm{a}}\right)$ mostró un buen ajuste. Sin embargo, las cargas factoriales del factor $F_{2}$ (Dedicación) fueron en general insustanciales $(<|.20|$, excepto el ítem 7; ver Tabla 3) y casi completamente absorbidas por el factor general (Fg). En vista de esto y de la aparente confirmación del reciente hallazgo de De Bruin y Henn (2013), se verificó el modelo bifactor incompleto $\left(\mathrm{M}_{3 \mathrm{~b}}\right)$, y se 
obtuvieron coeficientes de ajuste satisfactorios (ver Tabla 2). Los índices de modificación en este modelo también pusieron en evidencia que el modelo aun mejoraría luego de la liberación de los errores entre los ítems 9 y 10 , y 4 y 5 . Para procurar modificaciones parsimoniosas, se reespecificó el modelo bifactor incompleto $\left(\mathrm{M}_{3 \mathrm{c}}\right)$ con la covariación entre los ítems 9 y $10(r=.441, \mathrm{Cov}=.229, z=4.18, \mathrm{p}$ $<.01$ ), y se produjo un grado de ajuste comparativamente mejor que los modelos anteriores. La introducción del error correlacionado entre los ítems 9 y 10 produjo un impacto sustancial y más notorio sobre el cambio del RMSEA y AIC, similar al impacto de introducir más factores luego de evaluar el modelo unidimensional $\left(\mathrm{M}_{1}\right)$ y oblicuo $\left(\mathrm{M}_{2}\right)$.

Todas las cargas factoriales del modelo final $\left(\mathrm{M}_{3 \mathrm{c}}\right.$, bifactor incompleto) fueron estadísticamente significativos $(z>2.54)$ y las correspondientes con Fg fueron ligeramente mayores que las cargas en ambos factores específicos (Tabla 3). Por otro lado, los ítems que ahora únicamente obtenían su varianza de Fg mostraron sistemáticamente cargas elevadas. La evaluación final del modelo se hizo con la inspección del grado de sesgo de los parámetros en la introducción de un modelo bifactor (Reise, 2012; Reise et al., 2013). Se calcularon cuatro índices: el primero, la varianza común explicada (explained common variance, ECV; Reise et al., 2013), que ayuda a determinar la fuerza del factor general, y por lo tanto, unidimensionalidad. El ECV para el modelo bifactor completo $\left(\mathrm{M}_{3 \mathrm{a}}\right)$ e incompleto modificado $\left(\mathrm{M}_{3 \mathrm{c}}\right)$ fueron mayores a .70, el cual es un valor mínimo habitual para aceptar la unidimensionalidad (Reise et al., 2016a; Rodríguez et al., 2016b). En segundo lugar, la varianza común explicada en el nivel de los ítems (I-ECV; (Stucky, Thissen y Edelen, 2013), que debería ser alta. En la Tabla 3 (encabezado I-ECV), tiende a ser elevada, excepto para algunos ítems de Absorción (F3). Tercero, la proporción de correlaciones no contaminadas por la multidimensionalidad (PUC; Reise et al., 2013.) fue .724. Cuarto, los coeficientes de confiabilidad $\omega_{h}$ obtenidos (McDonald, 1999; Reise et al., 2013) para el factor general $\left(\omega_{h}>\right.$ $.80)$ y factores específicos $\left(\omega_{h}<.55\right)$ presentados en la Tabla 3 , indican un fuerte contraste entre ellas respecto a la varianza confiable. En conjunto, estos resultados señalan que el UWES-15 puede ser interpretado desde un marco de unidimensionalidad esencial (Rodríguez et al., 2016a, 2016b), e indica que la hipótesis de estudio se aceptaría. 
Tabla 3

Parámetros de los items en las soluciones factoriales del UWES-15.

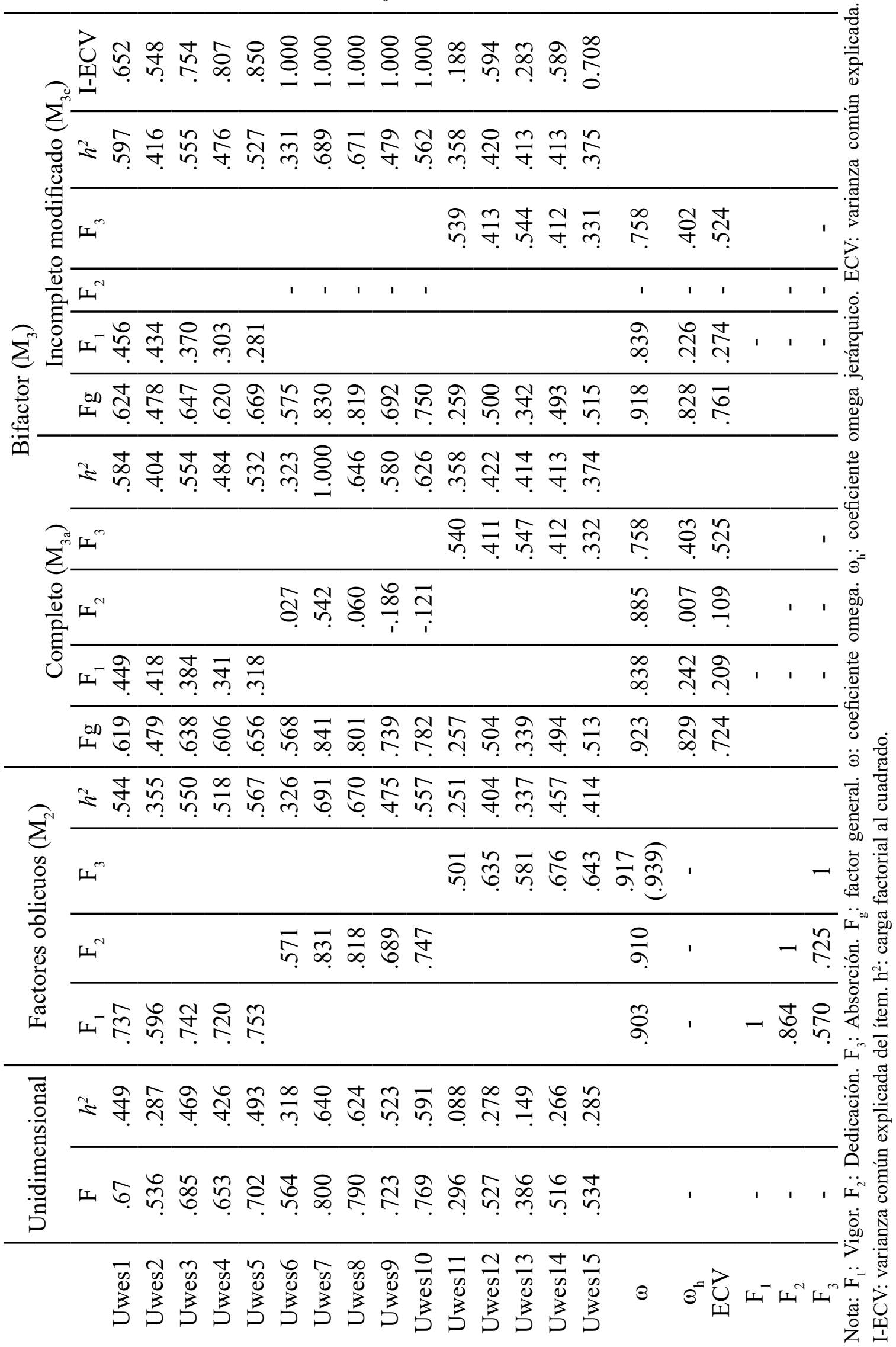




\section{UWES-9}

Una vez identificado el modelo de la versión completa, se examinó si este modelo era también apropiado para la versión de nueve ítems. Los resultados del ajuste para el modelo bifactor incompleto fueron buenos: SB- $\chi^{2}(\mathrm{gl}$ : $21)=30.954(p>.05)$, CFI $=.992$, RMSEA $($ IC $90 \%)=.027(.00, .047), \mathrm{SRMR}=.024, \mathrm{AIC}$ $=-11.046$; número de residuales $>|.10|=0$. No se observaron indicadores de modificación sustanciales entre los residuales de los ítems. El modelo bifactor completo también se examinó para propósitos de replicabilidad respecto al UWES-15, y se obtuvieron resultados similares con esta versión completa: $\mathrm{SB}-\chi^{2}(\mathrm{gl}: 18)$ $=28.555(\mathrm{p}>.05), \mathrm{CFI}=.992, \mathrm{RMSEA}(\mathrm{IC}$ $90 \%)=.030(.00, .050), \mathrm{SRMR}=.024, \mathrm{AIC}$ $=-7.444$; número de residuales $>|0.10|=0$. Por otro lado, el modelo unidimensional fue moderadamente satisfactorio: SB- $\chi^{2}$ (gl: 27) $=129.51(\mathrm{p}>.05), \mathrm{CFI}=.921, \mathrm{RMSEA}(\mathrm{IC}$ $90 \%)=.070(.06, .09), \mathrm{SRMR}=.065, \mathrm{AIC}=$ 75.51; número de residuales $>|0.10|=4$. Los resultados aquí obtenidos apuntan a la aceptación de la hipótesis de investigación.

Tabla 4

Modelos bifactor (completo e incompleto) para el UWES-9.

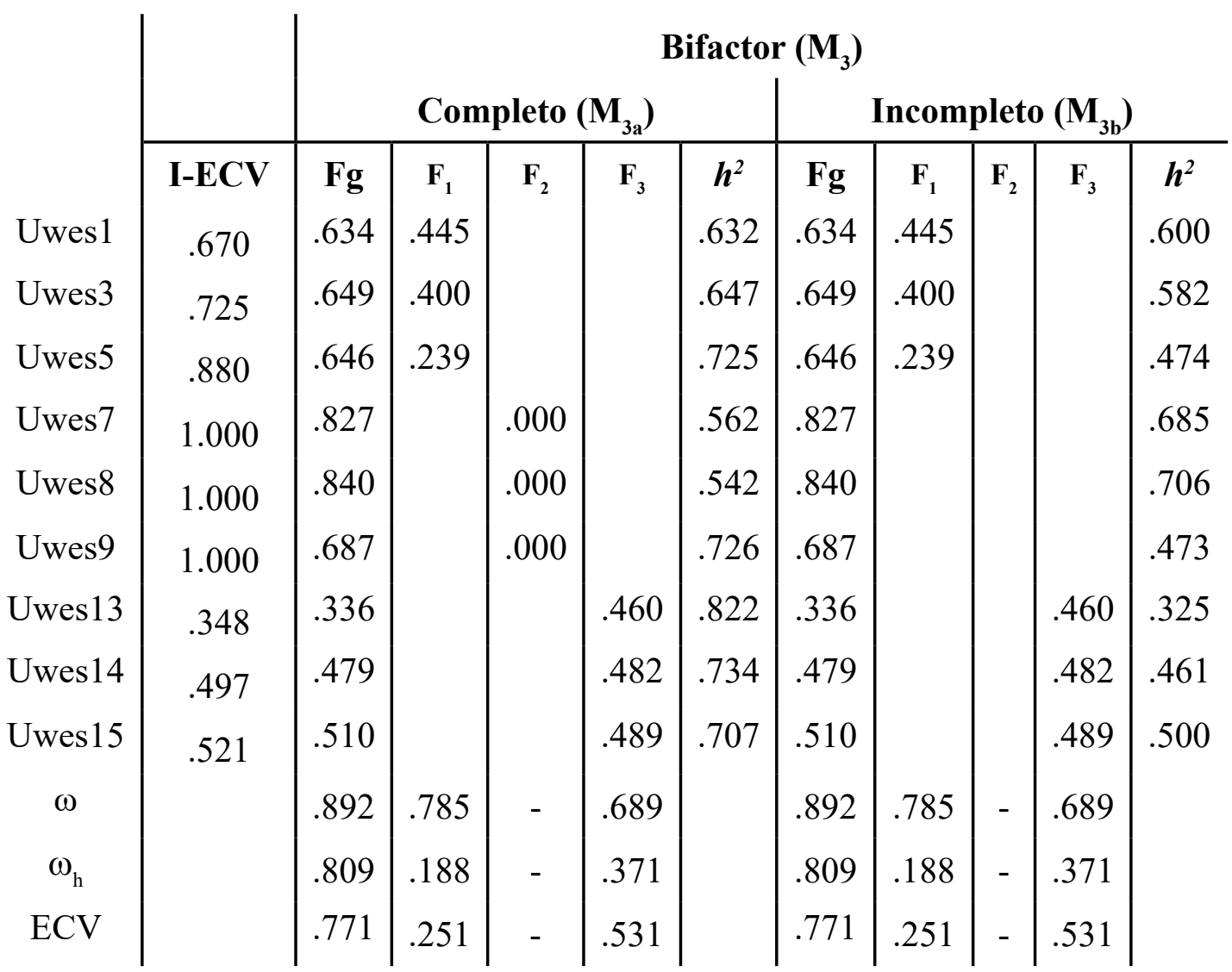

Notas: F1: Vigor. F2: Dedicación. F3: Absorción. Fg: factor general. $\omega$ : coeficiente omega. $\omega_{\mathrm{h}}$ : coeficiente omega jerárquico. ECV: varianza común explicada.

\section{Invarianza de medición}

Si los parámetros del modelo son estadísticamente equivalentes entre los grupos, la hipó- tesis de invarianza se mantiene entre los grupos comparados (docentes vs. no docentes). En la Tabla 5 se hallan los resultados de evaluación de IM, para ambas versiones del UWES. 
En la versión UWES-15, el ajuste de los modelos de base (configuracional) y constreñidos fueron satisfactorios, y la diferencia $\Delta_{\text {CFI }}$ entre los modelos comparados se mantuvieron debajo del criterio aplicado $(\leq .01$; Cheung y Resvold, 2002). Por lo tanto, las restricciones de igualdad impuestas a las cargas factoriales no degradaron sustancialmente los indicadores de ajuste, y por lo tanto, se satisfizo la invarianza métrica. En el siguiente nivel de invarianza, la aplicación de la igualdad de interceptos también arrojó cambios triviales en el CFI $\left(\Delta_{\mathrm{CFI}}<.01\right)$, lográndose también la invarianza fuerte. Por otro lado, las restricciones aplicadas consecutivamente a los ítems de la versión UWES-9 fueron también satisfactorias (Tabla 5), pues los índices de ajuste y $\Delta_{\text {CFI }}$ se mantuvieron debajo de los criterios elegidos. En resumen, la característica de IM se mantiene entre los grupos comparados, respecto a la configuración, discriminación de los ítems y escalamiento.

Tabla 5

Invarianza de medición del UWES-15 y UWES-9 (docentes vs. no docentes).

\begin{tabular}{c|c|c|c|c} 
& $\mathbf{S B}-\boldsymbol{\chi}^{\mathbf{2}}(\mathbf{g l})$ & $\mathbf{C F I}$ & $\Delta_{\text {CFI }}$ & RMSEA (IC 90 \%) \\
\hline UWES-15 & & & & \\
Configuracional & $291.423(158)$ & .950 & - & $.052(.042, .061)$ \\
Métrica & $339.591(183)$ & .941 & -.009 & $.052(.043, .060)$ \\
Escalar & $331.942(193)$ & .949 & .008 & $.044(.036, .051)$ \\
UWES-9 & & & & \\
Configuracional & $54.526(42)$ & .990 & - & $.031(.000, .052)$ \\
Métrica & $68.89(57)$ & .991 & .001 & $.026(.000, .045)$ \\
Escalar & $75.10(57)$ & .992 & .001 & $.032(.000, .050)$
\end{tabular}

Notas: $\Delta_{\mathrm{CFI}}$ : diferencia entre CFI

\section{Confiabilidad}

En la versión completa (15 ítems), la confiabilidad para la muestra total $\omega$ fue .883 (e.e. $=.008$, IC $95 \%$ : .865, .899); respecto a los grupos de trabajadores docentes $(\omega=$ .885 , e.e. $=.011$, IC $95 \%=.862, .908)$ y no docentes $(\omega=.871$, e.e. $=.014$, IC $95 \%=$ $.843, .899)$, se observó la similaridad entre ellos. En relación con el sexo, para varones fue $\omega=.865$ (e.e. $=.015$, IC $95 \%=.832$, .893 ) y para mujeres, $\omega=.897$ (e.e. $=.009$, IC $95 \%=.877, .914)$; en ambos, los coeficientes mostraron diferencias pequeñas. Las diferencias entre los grupos estimados no parecen ser sustancialmente diferentes. Para aplicar el coeficiente $\alpha$, primero se probó el ajuste de los datos al modelo tau-equivalente, con el método de Zhang y Yuan (2014); resultó que el modelo tau-equivalente no se ajustaba a los datos, tanto en la versión completa $[\mathrm{F}(102$, $532)=268.6, p<.01]$ como en la versión abreviada $[\mathrm{F}(35,601)=428.8, \mathrm{p}<.01]$. Para propósitos de comparación, fueron calculados los valores $\alpha$ de confiabilidad para la versión completa en la muestra total $(.887$, e.e. $=.005$, IC $95 \%=.875, .898)$, en varones $(.870$. e.e. $=$ .010 , IC $95 \%=.850, .890)$, en mujeres $(.898$, e.e. $=.006$, IC $95 \%=.885, .911)$, en docentes $(.888$, e.e. $=.007$, IC $95 \%=.873, .902) \mathrm{y}$ en no docentes $(.874$, e.e. $=.010$, IC $95 \%=.854$, .894). Estas estimaciones no difieren sustancialmente de las obtenidas con el coeficiente $\omega$. 
Por otro lado, para la versión abreviada (nueve ítems), en la muestra total se obtuvo una confiabilidad de $\omega=.851$ (e.e. $=.011$, IC $95 \%=.828, .874)$; en varones fue $\omega=$ .831 (e.e. $=.021$, IC $95 \%=.790, .872)$, y en mujeres, $\omega=.886$ (e.e. $=.013$, IC $95 \%=.839$, $.892)$. En el grupo de trabajadores docentes: $\omega=.858$ (e.e. $=.015$, IC $95 \%=.827, .888$ ), y en trabajadores no docentes: $\omega=.831$ (e.e. $=.020$, IC $95 \%=.791, .870)$. Como ocurrió con la versión larga, las diferencias entre los grupos parecen insustanciales. Cuando las estimaciones se hicieron con el coeficiente $\alpha$ en varones $(\alpha=.831$, e.e. $=.016$, IC $95 \%=$ $.800, .863)$, mujeres $(\alpha=.866$, e.e. $=.009, \mathrm{IC}$ $95 \%=.847, .885)$, docentes $(\alpha=.858$, e.e. $=$ .011 , IC $95 \%=.836, .880)$ y no docentes $(\alpha$ $=.831$, e.e. $=.015$, IC $95 \%=.799, .862)$, las diferencias entre ellas apenas fueron notorias, así como frente a las estimadas con el coeficiente $\omega$.

Los resultados obtenidos en el presente estudio se pueden resumir en lo siguiente: (a) el modelo de tres dimensiones del UWES no fue corroborado y una sola dimensión puede mejor representar la variabilidad de respuesta en el constructo medido; (b) la invarianza de medición se mantuvo respecto a los grupos ocupaciones comparados; y (c) la confiabilidad de consistencia interna fue alta para el puntaje total, en varios subgrupos y en la muestra total.

\section{Discusión}

La presente investigación encontró evidencias sobre la estructura interna del UWES-15 y UWES-9 en una muestra de trabajadores peruanos, que difiere de la forma en que generalmente ha sido tratado en estudios psicométricos del UWES en ambas versiones. Por este motivo, se planteó como objetivo examinar la estructura interna y se plantó la hipótesis sobre la dominancia de un factor general para la interpretación de los puntajes en el instrumento. En el estudio que se informa, la representación de tres factores correlacionados (u oblicuos) obtuvo un ajuste aceptable, lo cual es coherente con la teoría subyacente. Sin embargo, dada la inconsistencia de los resultados empíricos en estudios anteriores, esta estructura no parece ser la más satisfactoria, principalmente porque existe un problema asociado a la elevada covariación entre vigor, dedicación y absorción.

En el presente estudio, influenciado por trabajos relevantes (De Bruin y Henn, 2013; De Bruin, Hil, Henn y Muller, 2013; Fongy Ho, 2015; Tsubakita, Shimazaki, Ito y Kawazoe, 2017; Vallières et al., 2017), se encontró una posible forma estructural alternativa para expresar mejor las relaciones entre los ítems y los factores del UWES. De acuerdo a los antecedentes sobre el UWES, este podría ser utilizado desde un enfoque de dos niveles de interpretación (multidimensional y unidimensional). Sin embargo, se aplicaron herramientas diseñadas para evaluar directamente la multidimensionalidad (ECV, ECV-I, PUC, $\omega_{h}$, y $\left.\omega\right)$ y los resultados sugirieron otra forma interpretativa. Es decir, la incorporación del modelo bifactoral UWES representó el escenario típico, esto es, $\mathrm{PUC}<.80, \mathrm{ECV}>.60 \mathrm{y}$ $\omega_{\mathrm{h}}>$.70-0.80 (Reise et al., 2013; Rodríguez et al., 2016a, 2016b) para aceptar una solución unidimensional como la estructura preferida, en lugar de un modelo con dos niveles de interpretación (un factor general y factores específicos). Esta situación se acepta aun cuando la magnitud de los índices de ajuste práctico (e. g., RMSEA, CFI, TLI) obtenidos en el modelo unidimensional no son los mejores (Reise et al., 2013). Desde la arena metodológica, los índices que valoran la multidimensionalidad (ECV, ECV-I, PUC, $\omega_{h}, \mathrm{y} \omega$ ), generalmente, se consideran como la evidencia principal frente a los índices de ajuste en el CFA, aun cuando estos muestran estar moderadamente debajo de sus criterios habituales, por ejemplo, CFI < .94 (Reise et al., 2013).

Estos resultados están en la línea de dos vertientes de investigación: primero, en los estudios que aplicaron el modelamiento bifactor (De Bruin y Henn, 2013; De Bruin, 
Hil, Henn y Muller, 2013; Fongy Ho, 2015; Tsubakita et al., 2017; Vallières et al., 2017). También es usual respaldar un factor general, con suficiente varianza para representar la variación común de los ítems. Segundo, las correlaciones elevadas halladas también aquí también convergen con lo habitual, en investigaciones hispanas y no hispanas.

De esta situación emergen tres cuestiones que modifican la interpretación teórica original del UWES (que consiste en usar tres factores relacionados). El primero es la posible existencia de un factor general subyacente a los ítems que es estadísticamente sustancial como fuente de varianza de los ítems, independiente de factores específicos. En este factor general, los ítems contribuyen a su varianza, excepto dos cuya capacidad discriminativa es moderadamente baja (11 y 13, ambos del factor específico Absorción). El elemento principal de este factor general es el de Dedicación, cuyos ítems representaron completamente a este factor general. En segundo lugar, la diferenciación de dos factores específicos correspondientes a Vigor y Absorción parece no ser psicométricamente suficiente para describir constructos anidados dentro del constructo del engagement y, por lo tanto, podría cuestionarse su interpretación independiente del factor general. Sin embargo, un argumento contrario es que ambos factores muestran diferente monto de varianza específica, aunque bajos comparados con el factor general; por ejemplo, Absorción muestra más validez divergente comparado con Vigor, mientras que este contiene más varianza común (factor general). En tercer lugar, Dedicación fue absorbido completamente por el factor general y únicamente tiene importancia en la medida en que sus ítems se relacionan completamente con el factor general. Por lo tanto, este componente perdió autonomía estadística $\mathrm{y}$, consecuentemente, presenta una muy pobre diferenciación conceptual.

Con lo obtenido aquí, se puede concluir que la validez de la estructura interna de ambas versiones del UWES es parcialmente consis- tente con lo hallado por De Bruin y Henn (2013) porque, aunque ellos aceptaron la validez estructural de su factor general, también aceptaron los factores específicos. Pero, por otro lado, los resultados obtenidos en este estudio convergen con otros estudios, en los que la interpretación de un factor general del UWES es recomendable (De Bruin et al., 2013; Fong y Ho, 2015; Tsubakita, et al., 2017; Vallières et al., 2017). Efectivamente, en el presente estudio la fuerza del factor general, según los coeficientes especializados para detectarlo, es mayor debido al mayor monto de varianza retenida, lo que implica que sería conveniente operacionalizarlo mediante la construcción de un único puntaje observado. De acuerdo con la magnitud de las cargas factoriales, la replicabilidad del factor general puede ser mejor garantizada comparada con los factores específicos. Adicionalmente, aunque algunos de sus factores específicos retienen un monto de varianza no-común con el factor general, estos no muestran mejor definición psicométrica, suficiente fuerza y, potencialmente, son menos replicables. Teóricamente ello apuntaría a una concepción unidimensional para la interpretación del engagement, como de hecho los autores también lo han sugerido al obtener un puntaje único de la escala UWES (Schaufeli et al., 2006).

Como corolario de lo anterior, entre la versión de 15 o nueve ítems, sería más apropiado considerar la versión breve debido a que, en el UWES-15, una fuente adicional de variabilidad fue la liberación de errores de correlaciones entre los ítems de uno de los factores, mientras que en el UWES-9 no requirió incluir esto. La implicación de ello es que el UWES-9 tiene la ventaja adicional de la parsimoniosa representación de los constructos del engagement, sin fuentes residuales de varianza y psicométricamente invariante. Una versión aún más breve (tres ítems) fue recientemente desarrollada (Schaufeli et al., 2017), lo que parece respaldar la utilidad de usar formas reducidas del UWES. Efectivamente, ambas versiones son invariantes en sus 
propiedades psicométricas con respecto a la ocupación, lo que puede representar también la equivalencia entre ambas en docentes y no docentes. La invarianza de medición alcanzada sugiere que los ítems pueden funcionar similarmente en los diferentes niveles de puntuación (Elosua, 2011).

Algunas limitaciones del presente estudio deben ser mencionadas. Una de estas es que se trataron los ítems como variables continuas, lo cual generalmente se asocia a la violación de la normalidad multivariada (Lubke y Muthén, 2004); por lo tanto, estimados para variables categóricas son recomendados actualmente cuando el número de opciones, el grado de no normalidad y el tamaño muestral retienen la aplicación de métodos para variables tratadas como número continuos (Li, 2016; Muthén, du Toit y Spisic, 1997; Savalei y Rhemtulla, 2013). En contraste con esta limitación, los modelos de invarianza se ajustaron bien, y la incorporación de correcciones robustas al estadístico de ajuste tiende a ser eficiente (Savalei y Rhemtulla, 2013), sin comprometer errores de aceptación o el rechazo de los modelos. Otra limitación es que la representatividad de la muestra en relación con la población laboral no está garantizada y requiere que los resultados se corroboren en otros grupos poblacionales. Como balance para esta limitación, en los diferentes grupos participantes, en su composición y referente organizacional, los resultados fueron invariantes, lo que sugiere su generalización.

\section{Referencias}

Agarwal, U. A. (2014). Linking justice, trust and innovative work behaviour to work engagement. Personnel Review, 43, 41-73. https://doi. org/10.1108/PR-02-2012-0019

Bakker, A. B., Albrecht, S. L. y Leiter, M. P. (2011). Key questions regarding work engagement. European Journal of Work and Organizational Psychology, 20(1), 4-28. https://doi. org/10.1080/1359432X.2010.485352

Bentler, P. M. y Wu, E. J. C. (2012). EQS 6.2 for windows [Programa estadístico]. Encino, CA: Multivariate Software, Inc.

Berry, K. J. y Mielke, P. W., Jr. (1994). A test of significance for the Index of Ordinal Variation. Perceptual and Motor Skills, 79, 1291-1295.

Bollen, K. A. y Barb, K. H. (1981). Pearson's r and coarsely categorized measures. American Sociological Review, 46, 232-239. https://doi. org/10.2307/2094981

Cheung, G. W. y Rensvold, R. B. (2002). Evaluating goodness-of-fit indexes for testing measurement invariance. Structural Equation Modeling, 9(2), 233-255. https://doi. org/10.1207/s15328007sem0902_5

Christian, M. S. y Slaughter, J. E. (2007). Work engagement: A meta-analytic review and directions for research in an emerging area. Academy of Management Proceedings, 1, 1-6. https://doi.org/10.5465/ambpp.2007.26536346

Chughtai, A. A. y Buckley, F. (2013). Exploring the impact of trust on research scientists' work engagement. Personnel Review, 42, 396-421. https://doi.org/10.1108/PR-06-2011-0097

Cohen, J. (1992). A power primer. Psychological Bulletin, 112(1), 155-159. https://doi. org/10.1037/0033-2909.112.1.155

Colegio de Psicólogos del Perú. (2004). Código de Ética Profesional. http://www.fiapsi.org/ docs/etica/codigo_etico_peru.pdf

De Bruin, G. P. y Henn, C. M. (2013). Dimensionality of the 9-item Utrecht Work Engagement Scale (UWES-9). Psychological Reports, 112(3), 788-799. https://doi. org/10.2466/01.03.PR0.112.3.788-799

De Bruin, D. P., Hill, C., Henn, C. M. y Muller. K.P. (2013). Dimensionality of the UWES-17: An item response modelling analysis. South African Journal of Industrial Psychology, 39, 1-8. https://doi.org/10.4102/sajip.v39i2.1148

Doornik, J. A. y Hansen, H. (2008). An omnibus test for univariate and multivariate normality. Oxford Bulletin of Economics and Statistics, 70, 927-939. https://doi.org/10.1111/j.14680084.2008.00537.x

Dueber, D. M. (2017). Bifactor Indices Calculator: A Microsoft Excel-based tool to calculate various indices relevant to bifactor CFA 
models [computer program]. https://doi. org/10.13023/edp.tool.01

Elosua, P. (2011). Assessing measurement equivalence in ordered-categorical data. Psicológica, 32, 403-421.

Flores, C., Merino, C., Camacho, A., Juárez, A. y Placencia, O. (2015). Síndrome de burnout en instructores comunitarios: propiedades psicométricas del Maslach Burnout Inventory General Survey (MBI-GS). En A. JuárezGarcía (Coord.), Investigaciones psicométricas de escalas psicosociales en trabajadores mexicanos (pp. 279-308). Cuernavaca: UAEM / Plaza y Valdés Editores.

Fong, T. C. T. (2012). Measuring engagement at work: Validation of the Chinese Version of the Utrecht Work Engagement Scale. International Journal of Behavioral Medicine, 19, 391-397. http://doi.org/10.1007/s12529-011-9173-6

Fong, T. C. T. y Ho, R. T. H. (2015). Dimensionality of the 9-item Utrecht Work Engagement Scale revisited: A Bayesian structural equation modeling approach. Journal of Occupational Health, 57(4), 353-358. https://doi. org/10.1539/joh.15-0057-oa

Freeman J. B. y Dale R. (2013). Assessing bimodality to detect the presence of a dual cognitive process. Behavior Research Methods, 45, 83-97. https://doi.org/10.3758/s13428-0120225-x

Goldstein, R. (1996). sg59: Index of ordinal variation and Neyman-Barton GOF. Stata Technical Bulletin, 32, 10-11.

Hernández, C., Llorens, S. y Dickinson, M. (2016). Validación de la escala UWES-9 en profesionales de la salud en México. Pensamiento Psicológico, 14(2), 89-100. https://doi. org/10.11144/javerianacali.PPSI14-2.veup

Juárez-García, A., Vargas, C. I. H., Jiménez, C. A. F. y Ávila, A. C. (2015). Entusiasmo laboral en profesionales de la salud: Propiedades psicométricas de la Utrecht Work Engagement Scale. En A. Juárez-García (Ed.), Investigaciones Psicométricas de Escalas Psicosociales en Trabajadores Mexicanos (Capítulo 14). México, DF: Plaza y Valdés.

Kelley, K. y Pornprasertmanit, S. (2016). Confi- dence intervals for population reliability coefficients: Evaluation of methods, recommendations, and software for composite measures. Psychological Methods, 21(1), 69-92. https:// doi.org/10.1037/a0040086

Kelley, K. (2020). MBESS: The MBESS R Package. R package version 4.8.0. https:// CRAN.R-project.org/package=MBESS

Li, C. H. (2016). Confirmatory factor analysis with ordinal data: Comparing robust maximum likelihood and diagonally weighted least squares. Behavior Research, 48(3), 936-949. https:// doi.org/10.3758/s13428-015-0619-7

Lubke, G. H. y Muthén, B. O. (2004). Applying multigroup confirmatory factor models for continuous outcomes to Likert scale data complicates meaningful group comparisons. Structural Equation Modeling, 11(4), 514-534. https://doi.org/10.1207/s15328007sem1104_2

Malgady, R. G. (2007). How skewed are psychological data? A standardized index of effect size. Journal of General Psychology, 134(3), 355-359. https://doi.org/10.3200/ genp.134.3.355-360

Mardia, K. V. (1970). Measures of multivariate skewness and kurtosis with applications. Biometrika 57, 519-530. https://doi. org/10.2307/2334770

McDonald, R. P. (1999). Test theory: A unified approach. Mahwah, NJ: Erlbaum.

Meredith, W. (1993). MI, factor analysis and factorial invariance. Psychometrika, 58, 525-43. https://doi.org/10.1007/bf02294825

Müller, R., Pérez, C. y Ramírez, L. (2013). Estructura factorial y consistencia interna de la Utrecht Work Engagement Scale (UWES) 17 entre trabajadores sanitarios de Chile. Liberabit, 19(2), 163-171.

Muthén, B. O., du Toit, S. H. C. y Spisic, D. (1997). Robust inference using weighted least squares and quadratic estimating equations in latent variable modeling with categorical and continuous outcomes. http://gseis.ucla.edu/ faculty/muthen/articles/Article_075.pdf.

Petrović, I. B., Vukelić, M. y Čizmić, S. (2017). Work engagement in Serbia: Psychometric properties of the Serbian Version of the Utrecht 
Work Engagement Scale (UWES). Frontiers of Psychology, 16. https://doi.org/10.3389/ fpsyg.2017.01799

Reise, S. P., Scheines, R., Widaman, K. F. y Haviland, M. G. (2013). Multidimensionality and structural coefficient bias in structural equation modeling: A bifactor perspective. Educational and Psychological Measurement, 73, 5-26. https://doi. org/10.1177/0013164412449831

Reise, S. P. (2012). The rediscovery of bifactor measurement models. Multivariate Behavioral Research, 47(5), 667-696. https://doi.org/10.10 80/00273171.2012.715555

Rodríguez, A., Reise, S. P. y Haviland, M. G. (2016a). Evaluating bifactor models: Calculating and interpreting statistical indices. Psychological Methods, 21, 137-150. https:// doi.org/10.1037/met0000045

Rodríguez, A., Reise, S. P. y Haviland, M. G. (2016b). Applying bifactor statistical indices in the evaluation of psychological measures. Journal of Personality Assessment, 98, 223-237. https://doi.org/10.1080/00223891.20 15.1089249

Rodríguez-Montalbán, R., Martínez-Lugo, M. y Sánchez-Cardona, I. (2014). Análisis de las propiedades psicométricas de la Utrecht Work Engagement Scale en una muestra de trabajadores en Puerto Rico. Universitas Psychologica, 13(4), 1255-1266. http://doi. org/10.11144/Javeriana.UPSY13-4.appu

Salanova, M. y Llorens, S. (2008). Estado actual y retos futuros en el estudio del burnout. Papeles del Psicólogo, 29, 59-67.

Salanova, M., Schaufeli, W. B., Llorens, S., Peiró, J. M. y Grau, R. (2000). Desde el "burnout" al "engagement": ¿Una nueva perspectiva? Revista de Psicología del Trabajo y de las Organizaciones, 16, 117-134.

SAS Institute Inc. (2012). SAS/STAT 12.1 user's guide. Cary, NC: Author.

Satorra, A. y Bentler, P. M. (1994). Corrections to test statistics and standard errors in covariance structure analysis. En A. von Eye y C. C. Clogg (Eds.), Latent Variables Analysis: Applications for Developmental Research (pp. 399-419).
Thousand Oaks, CA: Sage.

Savalei, V. y Rhemtulla, M. (2013). The performance of robust test statistics with categorical data. The British Journal of Mathematical and Statistical Psychology, 66(2), 201-223. https:// doi.org/10.1111/j.2044-8317.2012.02049.x

Schaufeli, W. B. y Bakker, A. B. (2003). UWES-Utrecht work engagement scale: test manual. Unpublished Manuscript: Department of Psychology, Utrecht University, 8.

Schaufeli, W. B. y Bakker, A. B. (2010a). Defining and measuring work engagement: Bringing clarity concept. En A.B. Bakker y M.P. Leiter (Eds.), Work engagement: A handbook of essential theory and research (pp. 10-24). New York, NY: Psychology Press.

Schaufeli, W. B. y Bakker, A.B. (2010b). The conceptualization and measurement of work engagement. En A.B. Bakker y M.P. Leiter (Eds.), Work engagement: A handbook of essential theory and research (pp. 10-24), New York, NY: Psychology Press.

Schaufeli, W. B., Bakker, A. B. y Salanova, M. (2006). The measurement of work engagement with a short questionnaire: A cross-national study. Educational and Psychological Measurement, 66, 701-716. https://doi. org/10.1177/0013164405282471

Schaufeli, W. y De Witte, H. (2017). Work engagement: Real or redundant. Burnout Research, 5, 1-2. https://doi.org/10.1016/j. burn.2017.06.002

Schaufeli, W., Salanova, M., González-Romá, V. y Bakker, A. (2002). The measurement of burnout and engagement: A confirmatory factor analytic approach. Journal of Happiness Studies, 3, 71-92. https://doi. org/10.1023/A:1015630930326

Schaufeli, W., Shimazu, A., Hakanen, J., Salanova, M. y De Witte, H. (2017). An Ultra-short measure for Work Engagement. European Journal of Psychological Assessment. https:// doi.org/10.1027/1015-5759/a000430

Sinval, J., Pasian, S., Queirós, C. y Marôco, J. (2018). Brazil-Portugal transcultural adaptation of the UWES-9: Internal consistency, dimensionality, and measurement invari- 
ance. Frontiers of Psychology, 20. https://doi. org/10.3389/fpsyg.2018.00353

Sonnentag, S. (2003). Recovery, work engagement, and proactive behavior: A new look at the interface between non-work and work. Journal of Applied Psychology, 88, 518-528. https://doi.org/10.1037/0021-9010.88.3.518

Spontón, C., Medrano, L. A., Maffei, L., Spontón, M. y Castellano, E. (2012). Validación del cuestionario de Engagement UWES a la población de trabajadores de Córdoba, Argentina. Liberabit, 18(2), 147-154.

Spontón, C., Trógolo, M., Castellano, E. y Medrano, L. A. (2019). Medición del burnout: Estructura factorial, validez y confiabilidad en trabajadores argentinos. Interdisciplinaria, 36(1), 87-103. https://doi.org/10.16888/ interd.2019.36.1.7

Stucky, B. D., Thissen, D. y Edelen, M. O. (2013). Using logistic approximations of marginal trace lines to develop short assessments. Applied Psychological Measurement, 37, 23-39. https://doi.org/10.1177/0146621612462759

Tsubakita, T., Shimazaki, K., Ito, H. y Kawazoe, N. (2017). Item response theory analysis of the Utrecht Work Engagement Scale for Students (UWES-S) using a sample of Japanese university and college students majoring medical science, nursing, and natural science. BMC Research Notes, 10, 528. https://doi. org/10.1186/s13104-017-2839-7

United States. (1978). The Belmont report: Ethical principles and guidelines for the protection of human subjects of research. Bethesda, Md.: The Commission.

Vallières, F., McAuliffe, E., Hyland, P., Galligan, M. y Ghee, A. (2017). Measuring work engagement among community health workers in Sierra Leone: Validating the Utrecht Work Engagement Scale. Journal of Work and Organizational Psychology, 33, 41-46. https://doi. org/10.1016/j.rpto.2016.12.001

Vecina, M. L., Chacon, F., Sueiro, M. y Barron, A. (2012). Volunteer engagement: Does engagement predict the degree of satisfaction among new volunteers and the commitment of those who have been active longer? Applied Psychology: An International Review, 61, 130-140. https://doi.org/10.1111/j.14640597.2011.00460.x

Wefald, A. J. y Downey, R. G. (2009). Construct dimensionality of engagement and its relation with satisfaction. Journal of Psychology, 143, 91-111.

Zhang, Z. y Yuan, K.H. (2016). Robust coefficients alpha and omega and confidence intervals with outlying observations and missing data: methods and software. Educational and Psychological Measurement, 76(3), 387-411. https://doi.org/10.1177/0013164415594658

Recibido: 7 de abril de 2020 Aceptado: 25 de agosto de 2021 
Merino-Soto, Fernández-Arata y Juárez-García 